PROCEEDINGS OF THE

AMERICAN MATHEMATICAL SOCIETY

Volume 132, Number 2, Pages 543-551

S 0002-9939(03)07060-6

Article electronically published on June 12, 2003

\title{
AN EXISTENCE THEOREM OF HARMONIC FUNCTIONS WITH POLYNOMIAL GROWTH
}

\author{
YU DING \\ (Communicated by Richard A. Wentworth)
}

\begin{abstract}
We prove the existence of nonconstant harmonic functions with polynomial growth on manifolds with nonnegative Ricci curvature, Euclidean volume growth and unique tangent cone at infinity.
\end{abstract}

\section{INTRODUCTION}

For a noncompact, complete Riemannian manifold $\left(M^{n}, p\right)$ with nonnegative Ricci curvature,

$$
\operatorname{Ric}_{M^{n}} \geq 0
$$

we have the notion of tangent cone at infinity, which is any pointed GromovHausdorff limit of some sequence $M_{i}=\left(M^{n}, R_{i}^{-2} d x^{2}\right)$ with $R_{i} \rightarrow \infty$.

The almost rigidity theorem of Cheeger and Colding [4 implies that if $M^{n}$ has Euclidean volume growth, i.e., there is some $V_{\infty}>0$ such that for all $R>0$,

$$
\operatorname{Vol}\left(B_{R}(p)\right) \geq V_{\infty} R^{n}
$$

then every tangent cone at infinity is a metric cone, i.e., $\mathbf{R}_{+} \times X$ with the metric $d r^{2}+r^{2} d x^{2}$; here $\left(X, d x^{2}\right)$ is a metric space with diameter no more than $\pi$.

In this paper we will prove

Theorem 0.1. Assume that $M^{n}$ is a complete Riemannian manifold satisfying (0.1) and (0.2). Assume that $M$ has a unique tangent cone $C(X)$ at infinity. Then the dimension of the space of harmonic functions on $M^{n}$ with

$$
|u(y)| \leq C\left(1+d(p, y)^{N}\right)
$$

is at least $C\left(V_{\infty}\right) N^{n-1}$; here $C\left(V_{\infty}\right)>0$.

For each $N>0$, the space of harmonic functions $u$ with $(0.3)$ on manifolds with (0.1) is finite dimensional; this was conjectured by Yau and proved by Colding and Minicozzi in [11. See, for example, [12, 16] for further developments.

The tangent cone at infinity may not be unique; see [19], 5]. However, it is unique if we assume that the sectional curvature is nonnegative. Moreover, the example of Menguy [18] shows that even if $M^{n}$ has unique tangent cone, $M^{n}$ can have infinite topological type.

Received by the editors September 11, 2002 and, in revised form, October 8, 2002.

2000 Mathematics Subject Classification. Primary 53C21, 53C23.

(C)2003 American Mathematical Society 
By inspecting the proof of Theorem 0.1 we have, when the tangent cones are not unique,

Theorem 0.2. Assume that $M^{n}$ is a complete Riemannian manifold satisfying (0.1) and (0.2). Assume there exists $\lambda>0$, such that for all tangent cones $C(X)$, $\lambda$ is greater than $\lambda_{1}(X)$, the first eigenvalue of the Laplacian on $X$, and $\lambda$ is not an eigenvalue of any $X$. Then there exists a nonconstant polynomial growth harmonic function on $M^{n}$.

It seems the example in [5] satisfies the assumption above and so admits a nonconstant polynomial growth harmonic function.

There are manifolds that do not admit nonconstant harmonic functions with polynomial growth. For example, the manifold obtained by rounding off the end of $\mathbf{R}_{+} \times S^{n-1}$; one can check this directly or by [20]. Note this example satisfies ([0.1) but not (0.2).

In [13, the author showed that there is a separation of variables formula for the Laplacian on $C(X)$. In particular, there exist many harmonic functions on $C(X)$. We will transplant these harmonic functions back to balls on $M^{n}$; we then construct the desired harmonic functions by the Arzela-Ascoli theorem. In order to control the growth of these functions, we use a monotonicity Lemma 1.2, which is a generalization of the monotonicity of frequency for harmonic functions on $\mathbf{R}^{n}$ (see 1], 10], [9]).

Suppose that $\left(M_{i}^{n}, \operatorname{Vol}_{i}\right) \stackrel{d_{G H}}{\longrightarrow}\left(M_{\infty}, \mu_{\infty}\right)$ in the measured Gromov-Hausdorff sense, i.e., the sequence $\left\{M_{i}^{n}\right\}$ converges in the Gromov-Hausdorff sense to $M_{\infty}$, and for any $x_{i} \rightarrow x_{\infty}\left(x_{i} \in M_{i}^{n}\right)$ and $R>0$, we have $\operatorname{Vol}_{i}\left(B_{R}\left(x_{i}\right)\right) \rightarrow \mu_{\infty}\left(B_{R}\left(x_{\infty}\right)\right)$. In fact, for any sequences of manifolds with Ricci curvature bounded from below, after possible renormalization of the measures when $\left\{M_{i}^{n}\right\}$ is collapsing, there is a subsequence that converges in the measured Gromov-Hausdorff sense; moreover, under assumption (0.2), $\mu_{\infty}$ is just the $n$-Hausdorff measure on $M_{\infty}$. See [5].

Definition 0.3. Suppose $K_{i} \subset M_{i}^{n} \stackrel{d_{G H}}{\longrightarrow} K_{\infty} \subset M_{\infty}$ in the measured GromovHausdorff sense. $f_{i}$ is a function on $M_{i}^{n}, i=1,2, \ldots ; f_{\infty}$ is a continuous function on $M_{\infty}$. Assume that $\Phi_{i}: K_{\infty} \rightarrow K_{i}$ are $\epsilon_{i}$-Gromov-Hausdorff approximations, $\epsilon_{i} \rightarrow 0$. If $f_{i} \circ \Phi_{i}$ converge to $f_{\infty}$ uniformly, we say that $f_{i} \rightarrow f_{\infty}$ uniformly over $K_{i} \stackrel{d_{G H}}{\longrightarrow} K_{\infty}$.

For a Lipschitz function $f$ on $M_{\infty}$, one can define a norm

$$
\|f\|_{H_{1,2}}^{2}=\|f\|_{L^{2}}^{2}+\int_{M_{\infty}}|\operatorname{Lip} f|^{2}
$$

where $\operatorname{Lip} f$ is the pointwise Lipschitz constant

$$
\operatorname{Lip} f(x)=\limsup _{y \rightarrow x} \frac{|f(y)-f(x)|}{d(y, x)} .
$$

In [3], a Sobolev space $H_{1,2}$ is constructed by taking the closure of the norm (0.4). Moreover, one can define the differential $d f$ for $H_{1,2}$ functions $f$. In $[6$ it is proved that $M_{\infty}$ is $\mu_{\infty}$-rectifiable, and, as a corollary, (0.4) comes from an inner product $\langle\cdot, \cdot\rangle$. Thus $H_{1,2}$ transforms to a Hilbert space. Now by the standard theory of Dirichlet forms, one gets a positive self-adjoint Laplacian $\Delta$ on $M_{\infty}$,

$$
\int_{M_{\infty}}\langle d f, d g\rangle=\int_{M_{\infty}}(\Delta f) g
$$


see Theorem 6.25 of $[\underline{6}$.

The general philosophy is that the Laplacian $\Delta_{i}$ over $M_{i}$ "converge" to the operator $\Delta$ on $M_{\infty}$. We have the persistence of Poisson's equation [3], [6], [14]:

Lemma 0.4. Assume that $\Delta u_{i}=f_{i}$ on (a subset of) $M_{i}, \operatorname{Lip} u_{i}, \operatorname{Lip} f_{i} \leq L$ for some $L>0$. Assume that $u_{i} \rightarrow u_{\infty}, f_{i} \rightarrow f_{\infty}$ uniformly. Then on $M_{\infty}$ we have $\Delta u_{\infty}=f_{\infty}$.

We use some standard notation. Write

$$
f_{W} f=\frac{1}{\operatorname{Vol}(W)} \int_{W} f
$$

Denote by $A\left(p, R_{1}, R_{2}\right)$ the metric annulus $\left\{x \mid R_{1} \leq d(p, x) \leq R_{2}\right\}$. For any function $u_{i}$ we denote by $u_{i, p, R}$ the average of $u_{i}$ over $A(p, R / 2, R)$ :

$$
u_{i, p, R}=f_{A(p, R / 2, R)} u_{i} .
$$

The Laplacian operators are assumed to be positive.

After finishing this manuscript, Professor Colding pointed out to the author a paper of Zhang [22, in which nonconstant harmonic functions of polynomial growth can be constructed in the case when $C(X)$ is a smooth cone. Our construction turns out to be a generalization of [22] and applies to the case when $C(X)$ is not a smooth cone (so there are no coordinate systems available).

\section{AnAlysis ON METRIC CONES}

It is easy to see ([13]) that the $(n-1)$-Hausdorff measure on the cross section $X$ satisfies a doubling condition and the Poincare inequality. Moreover, the rectifiability as in [6] holds on $X$ as well; so one can define a Laplacian $\Delta_{X}$ on $X$. We have an eigenfunction expansion $\left\{\phi_{i}\right\}$ with $\Delta_{X} \phi_{i}=\lambda_{i} \phi_{i}$ on $X$. By the standard Moser iteration, the $\phi_{i}$ are Hölder continuous; later we will see that they are Lipschitz.

On a metric cone $C(X)$, there is a separation of variable formula [13]:

$$
\Delta u=-\frac{\partial^{2} u}{\partial r^{2}}-\frac{n-1}{r} \frac{\partial u}{\partial r}+\frac{1}{r^{2}} \Delta_{X} u .
$$

Therefore, if $\phi_{i}$ is the $i$-th eigenfunction of $\Delta_{X}$ on $X$ with eigenvalue $\lambda_{i}$, then $r^{\alpha_{i}} \phi_{i}(x)$ is harmonic; here $\alpha_{i}$ is the unique positive number with

$$
\lambda_{i}=\alpha_{i}\left(n+\alpha_{i}-2\right) .
$$

We normalize so that $\left\|\phi_{i}\right\|_{L^{2}(X)}=1$. Assume $u$ is harmonic on $B_{2}(p) \subset C(X)$. Then we can write (see [2], 8])

$$
u=\sum_{i=0}^{\infty} c_{i} r^{\alpha_{i}} \phi_{i}
$$

Define

$$
I(r)=\frac{1}{\operatorname{Vol}\left(\partial B_{r}\left(p_{\infty}\right)\right)} \int_{\partial B_{r}\left(p_{\infty}\right)} u^{2}
$$

here Vol is the $(n-1)$-Hausdorff measure; see [5]. $p_{\infty}$ is the pole of $C(X)$. Then

$$
I(r)=\sum_{i=0}^{\infty} c_{i}^{2} r^{2 \alpha_{i}} .
$$


Similarly to the Euclidean case ([14]), we have

Lemma 1.1. There is a $k>1$ that depends only on $X$ such that for $\epsilon>0$ sufficiently small, if $u$ is harmonic, then

$$
I(r) \leq\left(2^{\alpha_{1}+\epsilon}\right)^{2} I(r / 2)
$$

implies

$$
I(r / 2)<\left(2^{\alpha_{1}+\frac{\epsilon}{k}}\right)^{2} I(r / 4) .
$$

Proof. By (1.5), (1.6) is equivalent to

$$
\sum_{\alpha_{i} \neq \alpha_{1}} c_{i}^{2} r^{2 \alpha_{i}}\left(1-\frac{2^{2 \alpha_{1}+2 \epsilon}}{2^{2 \alpha_{i}}}\right) \leq \sum_{\alpha_{i}=\alpha_{1}} c_{i}^{2} r^{2 \alpha_{1}}\left(2^{2 \epsilon}-1\right) .
$$

On the other hand, (1.7) is equivalent to

$$
\sum_{\alpha_{i} \neq \alpha_{1}} \frac{1}{2^{2 \alpha_{i}}} c_{i}^{2} r^{2 \alpha_{i}}\left(1-\frac{2^{2 \alpha_{1}+\frac{2}{k} \epsilon}}{2^{2 \alpha_{i}}}\right) \leq \sum_{\alpha_{i}=\alpha_{1}} \frac{1}{2^{2 \alpha_{1}}} c_{i}^{2} r^{2 \alpha_{1}}\left(2^{\frac{2}{k} \epsilon}-1\right) .
$$

Thus, it suffices to show for $\alpha_{i} \neq \alpha_{1}$,

$$
\frac{1}{2^{2 \alpha_{i}}}\left(2^{2 \alpha_{i}}-2^{2 \alpha_{1}+\frac{2}{k} \epsilon}\right) /\left(2^{2 \alpha_{i}}-2^{2 \alpha_{1}+2 \epsilon}\right)<\frac{1}{2^{2 \alpha_{1}}}\left(2^{\frac{2}{k} \epsilon}-1\right) /\left(2^{2 \epsilon}-1\right) .
$$

Since there is a definite gap (that depends on $X$ ) between $\alpha_{1}$ and those $\alpha_{i} \neq \alpha_{1}$, the above holds when $k>1$ is sufficiently close to 1 and $\epsilon$ sufficiently small.

Corollary 1.11. Assume $u$ is harmonic. If

$$
f_{A\left(p_{\infty}, r / 2, r\right)} u^{2} \leq\left(2^{\alpha_{1}+\epsilon}\right)^{2} f_{A\left(p_{\infty}, r / 4, r / 2\right)} u^{2},
$$

then

$$
f_{A\left(p_{\infty}, r / 4, r / 2\right)} u^{2}<\left(2^{\alpha_{1}+\frac{\epsilon}{k}}\right)^{2} f_{A\left(p_{\infty}, r / 8, r / 4\right)} u^{2}
$$

Lemma 1.2. For $\epsilon$ small enough (as in Corollary 1.11), there exist $\delta, H>0, k>1$ depending only on $\epsilon$ such that if a manifold $(M, p)$ satisfies (0.1),

$$
d_{G H}\left(B_{4}(p), B_{4}\left(p_{\infty}\right)\right)<\delta
$$

$\left(B_{2}\left(p_{\infty}\right) \subset C(X)\right)$, then for any harmonic function u over $B_{2}(p)$, the inequality

$$
f_{A(p, 1 / 2,1)}\left|u-u_{p, 1}\right|^{2} \leq\left(2^{\alpha_{1}+\epsilon}\right)^{2} f_{A(p, 1 / 4,1 / 2)}\left|u-u_{p, 1 / 2}\right|^{2}
$$

implies

$$
f_{A(p, 1 / 4,1 / 2)}\left|u-u_{p, 1 / 2}\right|^{2}<\left(2^{\alpha_{1}+\frac{\epsilon}{k}}\right)^{2} f_{A(p, 1 / 8,1 / 4)}\left|u-u_{p, 1 / 4}\right|^{2}
$$

Proof. The proof is similar to the arguments in [14. Assume the lemma is not true; then for $\delta_{j} \rightarrow 0$, we can find a sequence of harmonic functions $u_{i}$ that satisfies (1.15) but not (1.16). After suitable renormalization, by the Cheng-Yau gradient estimate, a subsequence of $u_{i}$ will converge to a function $u_{\infty}$ on $C(X)$ satisfying (1.15) but not (1.16). Now by Lemma $0.4, u_{\infty}$ is harmonic, so we get a contradiction to Corollary 1.11 
Lemma 1.3. For all $\epsilon$ small enough, there exists $\delta$ such that if a manifold $(M, p)$ satisfies (0.1) and (0.2), and

$$
d_{G H}\left(B_{2}(p), B_{2}\left(p_{\infty}\right)\right)<\delta
$$

$\left(B_{2}\left(p_{\infty}\right) \subset C(X)\right)$, then for any nonconstant harmonic function u over $B_{2}(p)$,

$$
f_{A(p, 1 / 2,1)}\left|u-u_{p, 1}\right|^{2} \geq\left(2^{\alpha_{1}-\epsilon}\right)^{2} f_{A(p, 1 / 4,1 / 2)}\left|u-u_{p, 1 / 2}\right|^{2} .
$$

Proof. This is clearly true for harmonic functions on the metric cone $C(X)$. The proof follows from a compactness argument like the previous lemma.

Similarly, we have

Lemma 1.4. For $\epsilon<1$, there exist $\delta>0, k>1$ such that if a manifold $(M, p)$ satisfies (0.1) and (0.2), and

$$
d_{G H}\left(B_{4}(p), B_{4}\left(p_{\infty}\right)\right)<\delta
$$

$\left(B_{2}\left(p_{\infty}\right) \subset C(X)\right)$, then for any harmonic function $u$ over $B_{2}(p)$, the inequality

$$
\left|f_{A(p, 1,2)} u\right| \leq \epsilon\left(f_{A(p, 1,2)}|u|^{2}\right)^{\frac{1}{2}}
$$

implies

$$
\left|f_{A(p, 2,4)} u\right| \leq \frac{\epsilon}{k}\left(f_{A(p, 2,4)}|u|^{2}\right)^{\frac{1}{2}}
$$

\section{The BARrier AND APPlications}

Theorem 2.1. Assume $u_{\infty}$ is harmonic on the closed ball $B_{R}(p) \subset C(X)$. Then $u_{\infty}$ is the uniform limit of a sequence of harmonic functions $u_{i}$ on $B_{R}\left(p_{i}\right) \subset M_{i}$.

Proof. We approximate $\left.u_{\infty}\right|_{\partial B_{R}\left(p_{\infty}\right)}$ by Lipschitz functions, then by the transplantation theorem of Cheeger (Lemma 10.7 of [3]) we transplant it back to $M_{i}$ to a Lipschitz function $\beta_{i}$ on $\partial B_{R}\left(p_{i}\right) \subset M_{i}$,

$$
\left.\beta_{i} \rightarrow u_{\infty}\right|_{\partial B_{R}\left(p_{\infty}\right)} .
$$

Solve the Dirichlet problem

$$
\left\{\begin{array}{cl}
\Delta u_{i} & =0, \\
u_{i} & =\beta_{i}
\end{array} \quad \text { on } \quad \partial B_{R}\left(p_{i}\right) .\right.
$$

Since $M_{i} \stackrel{d_{G H}}{\longrightarrow} C(X)$, when $i$ is getting bigger we see the ball $B_{R}\left(p_{i}\right)$ almost satisfies an exterior sphere condition; see [15].

Fix $X_{\infty} \in \partial B_{R}\left(p_{\infty}\right)$. Pick $x_{i} \in \partial B_{R}\left(p_{i}\right)$ with $x_{i} \rightarrow x_{\infty}$. On the cone $C(X)$ there is a unique ray starting from the pole $p_{\infty}$, passing through $x_{\infty}$. Pick a point $q_{\infty}$ on this ray with $d\left(p_{\infty}, q_{\infty}\right)>d\left(p_{\infty}, x_{\infty}\right)$. Pick $q_{i} \in M_{i}$ with $q_{i} \rightarrow q_{\infty}$.

Consider $b_{i}(x)=d\left(q_{i}, x_{i}\right)^{2-n}-d\left(q_{i}, x\right)^{2-n}$. By the Laplacian comparison theorem,

$$
\Delta b_{i} \leq 0 .
$$


Thus exactly as in Chapter 2 of [15] we get two side bounds of $u_{i}$ near the boundary. Precisely, for all $\epsilon>0$ there exists $\delta$ such that for $x_{i} \in \partial B_{R}\left(p_{i}\right)$, $d\left(x, x_{i}\right) \leq \delta$ implies $\left|u_{i}(x)-u_{i}\left(x_{i}\right)\right| \leq \epsilon$, when $i$ is sufficiently large.

Now by the Arzela-Ascoli theorem, (a subsequence of) $u_{i}$ converges to some limit function $v_{\infty}$ on $C(X)$. By our estimate near the boundary and the maximum principle on $C(X),\left[3, v_{\infty}=u_{\infty}\right.$.

Note our argument does not imply that $u_{i}$ is continuous at the boundary.

By the Cheng-Yau gradient estimate we have

Corollary 2.4. Harmonic functions on $C(X)$ are Lipschitz. The eigenfunctions $\phi_{i}$ on $X$ are Lipschitz.

Corollary 2.5. The first eigenvalue $\lambda_{1}$ of $\Delta_{X}$ on $X$ satisfies $\lambda_{1} \geq n-1$.

Proof. The first eigenvalue $\lambda$ gives a harmonic function $r^{\alpha_{1}} \phi_{i}(x)$ on $C(X)$. Since it is Lipschitz, $\alpha_{1} \geq 1$. By (1.2) we have $\lambda_{1} \geq n-1$.

This is a generalization of the Lichnerowicz theorem. However, the Obata theorem does not hold: any $X$ such that $C(X)$ splits off some $\mathbf{R}$ satisfies $\lambda_{1}=n-1$.

\section{Proof of Theorem 0.1}

We now prove Theorem 0.1. Pick any sequence $R_{i} \rightarrow \infty$.

By the almost rigidity theorem of Cheeger-Colding [4, there exists a critical radius $R_{c}$ for $\alpha_{1}$ such that for all $r>R_{c}$, the assumptions of Lemma 1.2 Lemma 1.3 and Lemma 1.4, i.e., (0.1), (0.2), (1.14), hold on the rescaled manifold $\left(M^{n}, r^{-2} d x^{2}\right)$.

As in the previous section we transplant $u_{\infty}=r_{1}^{\alpha} \phi_{1}(x)$ back to harmonic functions $u_{i}$ on $B_{2}\left(p_{i}\right) \subset M_{i}=\left(M^{n}, R_{i}^{-2} d x^{2}\right)$ so that $u_{i} \rightarrow u_{\infty}$ uniformly.

We scale back and view $u_{i}$ as functions on $M^{n}$. By Theorem 2.1 for $R_{i}$ sufficiently large, at scale $R_{i}$ the harmonic function $u_{i}$ is close to some function $u_{\infty}=c r_{1}^{\alpha} \phi_{1}(x)$. Here and below, close means $L^{\infty}$-close, after an obvious rescale.

So, in particular, we can apply the monotonicity Lemma 1.2 in fact, we iterate it until the scale of critical radius $R_{c}$ when (the rescaled version of) (1.14) fails. So for all $R$ with $R_{c} \leq R \leq R_{i}$,

$$
f_{A(p, R / 2, R)}\left|u_{i}-u_{i, p, R}\right|^{2} \leq\left(2^{\alpha_{1}+\epsilon}\right)^{2} f_{A(p, R / 4, R / 2)}\left|u_{i}-u_{i, p, R / 2}\right|^{2} ;
$$

here recall $u_{i, p, R}$ is the average of $u_{i}$ on $A(p, R / 2, R)$.

Clearly $u_{i}$ is not a constant. We first subtract a constant and then multiply by a constant so that we can assume

$$
f_{A\left(p, R_{c} / 2, R_{c}\right)} u_{i}=0, \quad f_{A\left(p, R_{c} / 2, R_{c}\right)} u_{i}^{2}=1
$$

So by iterating Lemma 1.4, for all $R$ with $R_{c} \leq R \leq R_{i}$,

$$
\left|u_{i, p, R}\right|=\left|f_{A(p, R, R)} u_{i}\right| \leq \epsilon\left(f_{A(p, R / 2, R)} u_{i}^{2}\right)^{1 / 2} .
$$


We have

$$
\begin{aligned}
\left(f_{A\left(p, R_{c}, 2 R_{c}\right)} u_{i}^{2}\right)^{1 / 2} & \leq\left(f_{A\left(p, R_{c}, 2 R_{c}\right)}\left|u_{i}-u_{i, 2 R_{c}}\right|^{2}\right)^{1 / 2}+\left|u_{i, 2 R_{c}}\right| \\
& \leq 2^{\alpha_{1}+\epsilon}\left(f_{A\left(p, R_{c} / 2, R_{c}\right)} u_{i}^{2}\right)^{1 / 2}+\epsilon 2^{\alpha_{1}+\epsilon}\left(f_{A\left(p, R_{c} / 2, R_{c}\right)} u_{i}^{2}\right)^{1 / 2} \\
& \leq 2^{\alpha_{1}+2 \epsilon}\left(f_{A\left(p, R_{c} / 2, R_{c}\right)} u_{i}^{2}\right)^{1 / 2} .
\end{aligned}
$$

Iterating this, we have

$$
\left(f_{A\left(p, 2^{j-1} R_{c}, 2^{j} R_{c}\right)} u_{i}^{2}\right)^{1 / 2} \leq 2^{\left(\alpha_{1}+2 \epsilon\right) j}\left(f_{A\left(p, R_{c} / 2, R_{c}\right)} u_{i}^{2}\right)^{1 / 2}
$$

So $u_{i}$ (defined on $B_{R_{i}}(p)$, with $R_{i} \gg R_{c}$ ) is of polynomial growth,

$$
\left|u_{i}\right| \leq C r^{\alpha_{1}+2 \epsilon} \text {. }
$$

Combining with the Cheng-Yau gradient and the Arzela-Ascoli theorem, $u_{i}$ converges to a nonconstant polynomial growth harmonic function $u^{(1)}$ on $M$.

Next, we indicate how to construct a second harmonic function when there is another eigenfunction for $\lambda_{1}$. By construction, $u^{(1)}$ satisfies (3.1) and (3.3) at every scale $R>R_{c}$. So by Lemma 0.4 on any sufficiently large scale, $u^{(1)}$ is close to a function of the form

$$
\sum_{\alpha_{i}=\alpha_{1}} c_{i} r^{\alpha_{1}} \phi_{i}(x)
$$

on $C(X)$. Note that we have no control over the constants $c_{i}$. By assumption, $\lambda_{1}$ has more than one multiple; so there is a function of the form

$$
\sum_{\alpha_{i}=\alpha_{1}} b_{i} r^{\alpha_{1}} \phi_{i}(x)
$$

that is perpendicular to (3.7) on $C(X)$. Like the construction of $u^{(1)}$, we transplant (3.8) back to $M_{i}$, solve the Dirichlet problem as in (2.2), and get a sequence of harmonic functions $w_{i}^{(2)}$. Now adjust $w_{i}^{(2)}$ by a tiny constant, then subtract $c u^{(1)}$, a multiple of our first harmonic function $u^{(1)}$, so that

$$
u_{i}^{(2)}:=\left(w_{i}^{(2)}-c u^{(1)}\right) \perp u^{(1)} \quad \text { on } \quad A\left(p, R_{c}, 2 R_{c}\right) .
$$

Note that we have no control over the constant $c$, but this is not important since all we need is that on scale $R_{i}$ we have the inequality (3.1), and $u_{i}^{(2)}$ is not a constant. Then as before we construct our second function $u^{(2)}$. It is independent of $u^{(1)}$ since it is perpendicular to $u^{(1)}$ on $u^{(1)} A\left(p, R_{c}, 2 R_{c}\right)$.

The constructions of all the other harmonic functions follow the same pattern. Note then we need a revised version of Lemma 1.2 in which $\alpha_{1}$ is substituted by $\alpha_{i}$. The generalization is straightforward.

Clearly, if we have $N$ eigenvalues of $X$ with $\lambda \leq \Lambda=N(N+n-2)$, then we have at least $N$ independent nonconstant harmonic functions $u^{(j)}$ with

$$
\left|u^{(j)}(y)\right| \leq C(j, \epsilon)\left(1+d(p, y)^{N+\epsilon}\right) .
$$


Now we can count them. By a well-known argument in estimating upper bounds of eigenvalues (similar to p. 105 of [21]), we have

$$
\lambda_{j} \leq C(n)\left(\frac{j}{H^{n-1}(X)}\right)^{\frac{2}{n-1}}
$$

here $H^{n-1}(X)$ is the $(n-1)$-Hausdorff measure of $X$. Actually, we can take $V_{\infty}$ in (0.2) for it; see [5]. So there are at least $C\left(V_{\infty}\right) \Lambda^{\frac{n-1}{2}}$ many eigenvalues less than $\Lambda$, and the dimension of harmonic functions with

$$
|u(y)| \leq C\left(1+d(p, y)^{N}\right)
$$

is at least $C\left(V_{\infty}\right) N^{n-1}$.

Finally, we remark that the technical assumption in Theorem 0.2 is needed to guarantee that Lemma 1.2 works when $C(X)$ is not unique.

\section{REFERENCES}

[1] F. Almgren, Dirichlet's problem for multiple valued functions and the regularity of mass minimizing integral currents. Minimal submanifolds and geodesics (Proc. Japan-United States Sem., Tokyo, 1977), pp. 1-6, North-Holland, Amsterdam, New York, 1979. MR 82g:49038

[2] J. Cheeger, Spectral geometry of singular Riemannian spaces. J. Differential Geom. 18 (1983), 575-657. MR 85d:58083

[3] J. Cheeger, Differentiability of Lipschitz functions on metric measure spaces, Geom. Funct. Anal. 9 (1999) 428-517. MR 2000g:53043

[4] J. Cheeger and T. Colding, Lower bounds on Ricci curvature and the almost rigidity of warped products, Ann. of Math. (2) 144 (1996), no. 1, 189-237. MR 97h:53038

[5] J. Cheeger and T. Colding, On the structure of spaces with Ricci curvature bounded below, Part I. J. Differential Geom. 46 (1997), no. 3 406-480. MR 98k:53044

[6] J. Cheeger and T. Colding, On the structure of spaces with Ricci curvature bounded below, Part 3. J. Differential Geom. 54 (2000), no. 1, 37-74. MR 2003a:53044

[7] J. Cheeger, T. Colding, and W. Minicozzi II, Linear growth harmonic functions on complete manifolds with nonnegative Ricci curvature, Geom. Funct. Anal. 5 (1995), no. 6, 948-954. MR 96j:53038

[8] J. Cheeger and M. Taylor, On the diffraction of waves by conical singularities. I. Comm. Pure Appl. Math. 35 (1982), no. 3, 275-331. MR 84h:35091a

[9] J. Cheeger and G. Tian, On the cone structure at infinity of Ricci flat manifolds with Euclidean volume growth and quadratic curvature decay. Invent. Math. 118 (1994), no. 3, 493571. MR 95m:53051

[10] T. Colding and W. Minicozzi II, Harmonic functions with polynomial growth. J. Differential Geom. 46 (1997), no. 1, 1-77. MR 98h:53060

[11] T. Colding and W. Minicozzi II, Harmonic functions on manifolds. Ann. of Math. (2) 146 (1997), no. 3, 725-747. MR 98m:53052

[12] T. Colding and W. Minicozzi II, Liouville theorems for harmonic sections and applications. Comm. Pure Appl. Math. 51 (1998), no. 2, 113-138. MR 98m:53053

[13] Y. Ding, Heat kernels and Green's functions on limit spaces. Comm. Anal. Geom. 10 (2002), no. $3,475-514$

[14] Y. Ding, The gradient of certain harmonic functions on manifolds of almost nonnegative Ricci curvature. Israel J. Math. 129 (2002), 241-251.

[15] D. Gilbarg and N. Trudinger Elliptic Partial Differential Equations of Second Order, Grundlehren der mathematischen Wissenschaften, Vol. 224, Springer-Verlag, New York, 1983. MR 86c:35035

[16] P. Li, Harmonic sections of polynomial growth. Math. Research Letters, Vol. 4 (1997), 35-44. MR 98i:53054

[17] F. Lin, Asymptotically conic elliptic operators and Liouville type theorems. Geometric analysis and the calculus of variations, 217-238, Internat. Press, Cambridge, MA, 1996. MR 2000c:35051 
[18] X. Menguy, Noncollapsing examples with positive Ricci curvature and infinite topological type Geom. Funct. Anal. 10 (2000), no. 3, 600-627. MR 2001g:53074

[19] G. Perelman, A complete Riemannian manifold of positive Ricci curvature with Euclidean volume growth and nonunique asymptotic cone. Comparison geometry, 165-166, Cambridge Univ. Press, Cambridge, 1997. MR 98e:53067

[20] C. Sormani, Harmonic functions on manifolds with nonnegative Ricci curvature and linear volume growth. Pacific J. Math. 192 (2000), no. 1, 183-189. MR 2001e:53034

[21] R. Schoen, S-T. Yau, Lectures on Differential Geometry. International Press, Cambridge, MA, 1994. MR 97d:53001

[22] L. Zhang, On the generic eigenvalue flow of a family of metrics and its application. Comm. Anal. Geom. 7 (1999), no. 2, 259-278. MR 2000f:53053

Department of Mathematics, University of California, Irvine, California 92697

E-mail address: yding@math.uci.edu 